\title{
Low-power programmable linear-phase filter designed for fully balanced bio-signal recording application
}

\author{
Guohe Zhang, Huibin Tao ${ }^{\text {a) }}$, Jun Shao, Shaochong Lei, \\ and Feng Liang
}

School of Electronic and Information Engineering, Xi'an Jiaotong University, Xi'an, China, 710049.

a) coldfire_arm@mail.xjtu.edu.cn

\begin{abstract}
A reconfigurable fully differential programmable linearphase filter is presented in the paper which can be used in portable multichannel electroencephalogram, electrocardiogram, wireless brainheart monitoring systems used in hospital or home care settings. To reduce the influence of coefficient sensitivity and maintain an undistorted bio-signal, a fifth-order ladder-type Bessel Transconductance-Capacitor (Gm-C) lowpass filter is employed. The filter is designed as part of on-chip self-adjusting noise suppression block and its programmability makes it suitable for different biomedical signals processing. Simulation results show that this low-voltage and low-power filter possesses the HD3 of $50.7 \mathrm{~dB}$, dynamic range of $62 \mathrm{~dB}$, dc gain of $-2.899 \mathrm{~dB}$, and power consumption of $30 \mu \mathrm{W}$. Meanwhile, the designed filter can effectively reduce self-adjusting noise on specific channels and anti-aliasing of the analog-to-digital converter (ADC).
\end{abstract}

Keywords: linear-phase filter, programmable, Gm-C lowpass filter, bio-signal

Classification: Integrated circuits

\section{References}

[1] B. Gosselin, M. Sawan, and C. A. Chapman, "A Low-Power Integrated Bioamplifier With Active Low-Frequency Suppression," IEEE Trans. Biomed. Circuits Syst., vol. 1, pp. 184-192, Sept. 2007.

[2] W. Sansen and P. M. Van Peteghem, "An area-efficient approach to the design of very-large time constants in switched-capacitor integrators," IEEE J. Solid-State Circuits, vol. SC-19, pp. 772-780, Oct. 1984.

[3] E. Rodriguez-Villegas, A. Yufera, and A. Rueda, "A 1.25-V micropower Gm-C filter based on FGMOS transistors operating in weak inversion," IEEE J. Solid-State Circuits, vol. 39, no. 1, pp. 100-111, Jan. 2004.

[4] X. Qian, Y. P. Xu, and X. Li, "A CMOS continuous-time lowpass notch filter for EEG systems," Analog Integr. Circuits Signal Process., vol. 44, pp. 231-238, July 2005.

[5] K. Hyung-Ock and S. Youngsoo, "Semicustom design methodology of power gated circuits for low leakage applications," IEEE Trans. Circuits 
Syst. II, Exp. Briefs, vol. 54, no. 6, pp. 512-516, June 2007.

[6] S. Y. Lee and C. J. Cheng, "Systematic Design and Modeling of a OTA-C Filter for Portable ECG Detection," IEEE Trans. Biomed. Circuits Syst., vol. 3, pp. 53-64, Feb. 2009.

\section{Introduction}

Low-frequency filters are important building blocks in analog front-end system, which are adopted to decrease the out-of-band noise. However, for low frequency biomedical applications, realizing lowpass filter circuits with large time constant under an acceptable capacitor's value isn't an easy task [1]. Switched capacitor (SC) techniques [2] have been preferred over continuoustime design techniques (MOSFET-C and active RC filters). This is mainly due to their high accuracy, low sensitivity to parasitic capacitors, and reduced harmonic distortion components. However, SC implementations require precise on-board clock and phase generators. Moreover, for very large time constants, large capacitor ratios are mandatory. There have been the proposal to use low-power continuous-time well-suited Operational Transconductance Amplifier (OTA) based filters composed of the open-loop Gm-C integrators $[3,4,5,6]$ to realize a very low transconductance (Gm, typically of the order of nanoamperes per volt). In OTA-based circuits, the OTA will dominate the performance of the filter circuit, and the ratio of the capacitor to the small transconductance determines the time constant of Gm-C integrators.

Taking consideration of the affections of dynamic range, noise level, power dissipation, and silicon area constraints, this paper presents a fifth-order GmC lowpass filter with very small transconductance. This biomedical filter can reduce out-of-band noise on specific channels and anti-aliasing of the analogto-digital converter (ADC).

\section{Biomedical filter design}

In portable or implantable biomedical systems where power consumption is of primary concern, it is necessary to reduce the number of active building blocks and maximize the power efficiencies of each block. In order to precisely record the bio-signal, the detection circuits must be capable of attenuating the outof-band interference and the noise before ADC to avoid the aliasing. For this reason, a fifth-order Bessel filter with a minimum phase distortion and three low cutoff frequencies for different biomedical signals processing are chosen for this design. A good enough inband performance will be achieved for the filter to detect biomedical signal precisely. The challenge is to design amplifiers with very small transconductance for a Gm-C filter under constraints with dynamic range, noise level, power dissipation, and silicon area. 


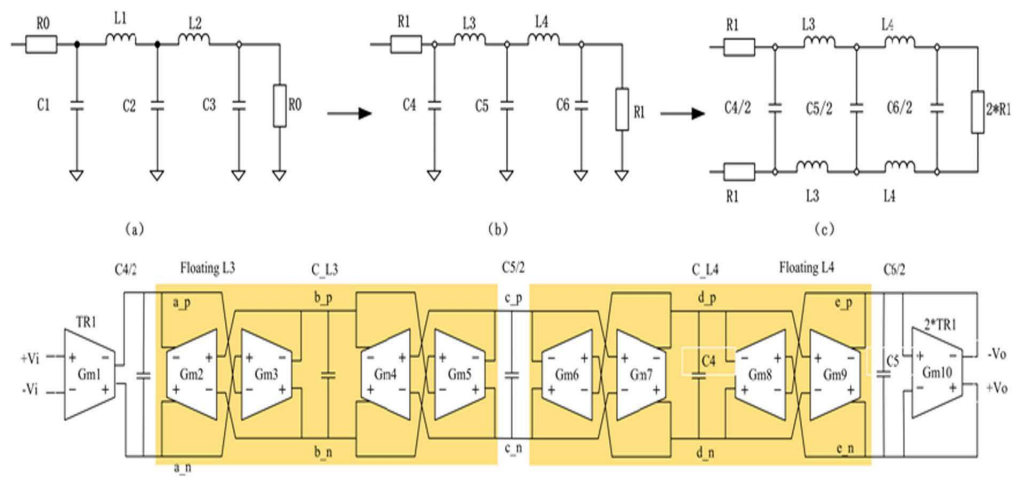

Fig. 1. Fifth-order low-pass filter model (a) Normalized at $1 \mathrm{rad} / \mathrm{sec}$ cutoff frequency (b) Single-ended scaled model (c) Fully differential scaled model (d) Active circuit realization of fifth-order Bessel filter

\subsection{Filter synthesis}

A normalized fifth-order passive ladder-type Bessel filter at $1 \mathrm{rad} / \mathrm{sec}$ cutoff frequency is deduced as shown in Fig. 1(a). Based on the topology, a corresponding circuit with the scaled coefficients is depicted in Fig. 1 (b). If $\mathrm{R} 1=\mathrm{aR} 0$ in Fig. 1 (b) and Fig. 1 (c), the scaled capacitor and inductance can be calculated as

$$
\begin{aligned}
& C_{\text {scaled }}=C_{\text {normalized }} / \alpha M \\
& L_{\text {scaled }}=\alpha L_{\text {normalized }} / M
\end{aligned}
$$

Here, $M=2 \pi B W$ and BW is the geometry center $-3 \mathrm{~dB}$ cutoff frequency. The normalized low-pass filter model can be used to realize any frequency filter.

Moreover, fully differential circuits are chosen due to a higher commonmode rejection and an increment of $3 \mathrm{~dB}$ in dynamic range rather than singleend structure. Based on the topology, a corresponding active circuit with the scaled coefficients, as depicted in Fig. 1 (d), can be carried out through substituting the OTAs for these passive components.

\subsection{Frequency programmable}

For a filter, the unity-gain frequency is proportional to the product of its transconductance and its loading capacitor. Changing either transconductance or loading capacitor can change the unity-gain frequency proportionally. This property is called frequency programmability. In our design, a switch array is used in the Gm-C filter design to realize three low cutoff frequencies for different biomedical signals processing, that is, $35 \mathrm{~Hz}$ for heart rate detection, $150 \mathrm{~Hz}$ for EEG signal detection and $230 \mathrm{~Hz}$ for ECG signal detection. Five capacitor arrays (C4/2, C_L3, C5/2, C_L4, C6/2) are designed to realize adjustable programmable bandwidths. To further save area, the capacitor array and logic circuitry are designed so that for every two adjacent bandwidths, the lower bandwidth (bigger capacitor) uses all capacitors 


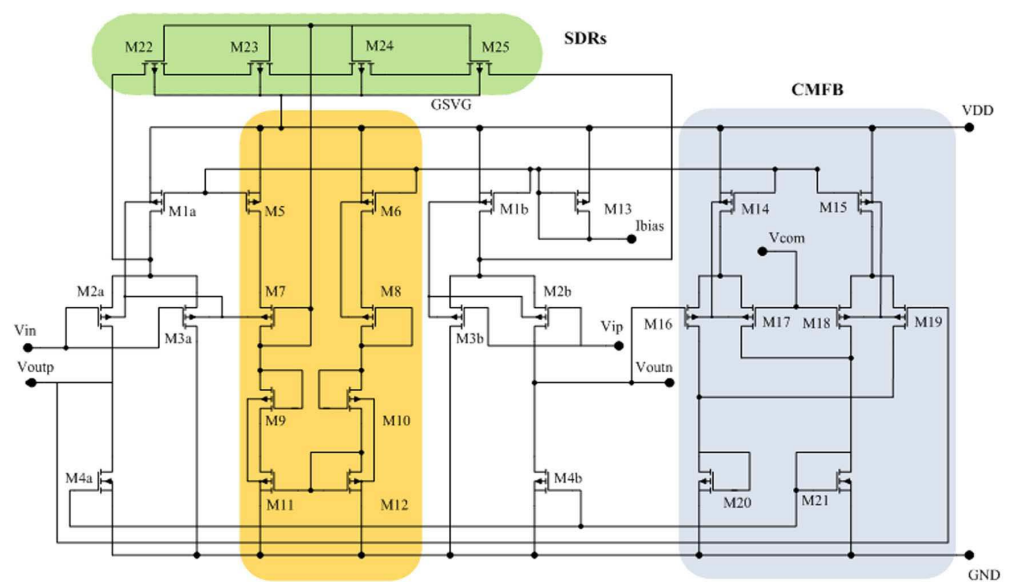

Fig. 2. Operational transconductance amplifier

while the higher bandwidth uses a part of the capacitors.

In the Gm-C filter implementation, the transconductors within the circuit have the gradually decreasing values of transconductance: $2.65 \mathrm{nS}, 1.60 \mathrm{nS}$, and $0.60 \mathrm{nS}$. That is, $\mathrm{Gm} 1=\mathrm{Gm} 2=\mathrm{Gm} 3=\mathrm{Gm} 4=\mathrm{Gm} 5=2.65 \mathrm{nS}, \mathrm{Gm} 6=\mathrm{Gm} 7=$ $\mathrm{Gm} 8=\mathrm{Gm} 9=1.60 \mathrm{nS}$, and $\mathrm{Gm} 10=0.60 \mathrm{nS}$. The increased impedance will reduce the losses of the dc gain. On the other hand, for a given pole/zero frequency, the value of $\mathrm{gm}$ is proportional to the capacitor value. Thus, smaller transconductance mean smaller capacitors and smaller bias currents. This provides a more robust design keeping bias currents in the nanoamp range.

\subsection{Linearized OTA design}

In OTA-based filters, voltage-to-current converters can generate huge harmonic distortion components; hence it is mandatory to employ linearized OTAs. The OTA used in this paper, shown in Fig. 2, combines these techniques: inversion and triode region is split into four transistors (M22, M23, M24, and M25) and their source-gate voltage is controlled by the self-biased transistor M7 with constant biased current. As a result, the small signal transconductance is marginally sensitive to the common-mode input voltage. Then the differential input voltage (Vin-Vip) is converted to current by source-degeneration transistors M22, M23, M24, and M25 which are operated in triode region. Transistors M2a and M3a split the drain current of sourcedegeneration transistors. Most of the current flows to ground through transistors M3a because we designed the dimensions so that $g_{m, M 2 a}>>g_{m, M 3 a}$.

\section{Results discussion}

The proposed biomedical filter was implemented with SMIC $0.18 \mu \mathrm{m}$ CMOS technology, and the power supply is $1.8 \mathrm{~V}$. In order to decrease the mismatch of transconductance and the parasitic of wiring, the Gm cells are placed as closely as possible to each other. Since the biomedical monitoring system is often with a programmable low-noise instrumentation amplifier prior to the antialias filter, the amplitude of the weak bioelectric signal will be am- 
plified to a higher level before further processing. Therefore, the following simulations are made, under the assumption that the signal source had been amplified to the maximum input range of the filter with negligible noise and interference.

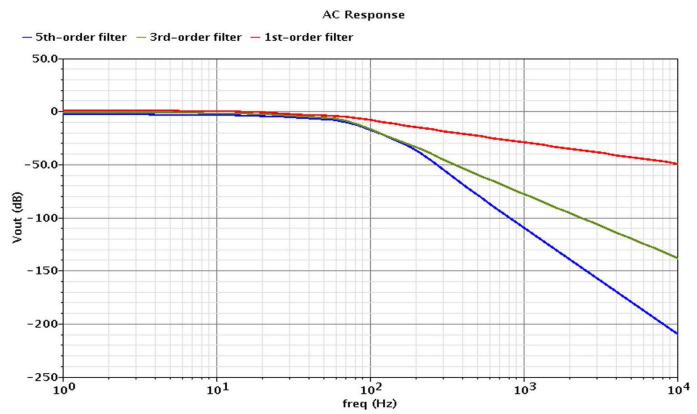

(a)

AC Response

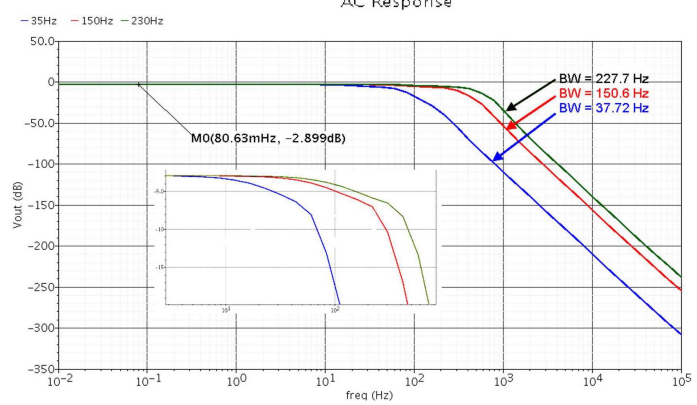

(b)

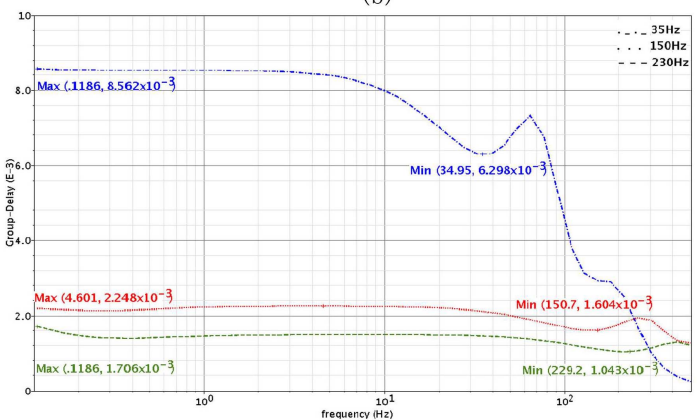

(c)

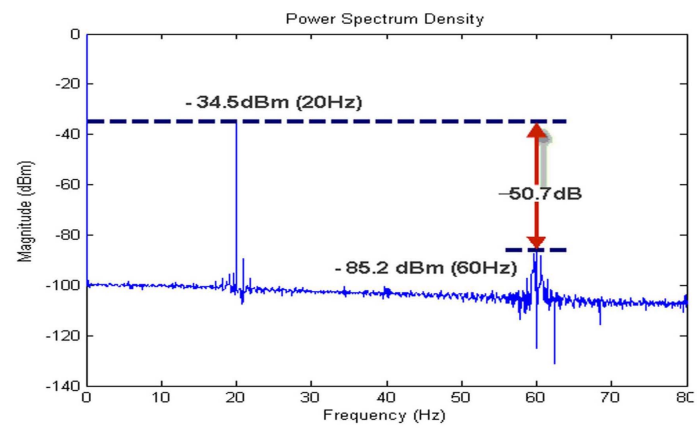

(d)

Fig. 3. (a) 1st, 3rd, 5th-order filter with different attenuation in the stopband, (b) Frequency response of the fifth-order Gm-C filter, (c) Group delay of the fifth-order Gm-C filter, (d) Measured output power spectrum of the low-pass filter with a sinusoidal input signal of $20 \mathrm{~Hz}$ and $200 \mathrm{mVpp}$ 
Fig. 3 (a) depicted the 1st, 3rd, 5th-order filter with different attenuation in the stopband, which is obtained by controlling switch teams. This explains the designed filter can effectively reduce self-adjusting noise indirectly. Then the fifth-order ladder-type Bessel Gm-C lowpass filter is simulated under stress conditions. Fig. 3 (b) shows the simulated magnitude response of the proposed filter, the cutoff frequency of the fifth-order low-pass filter is set to $37.7 \mathrm{~Hz}, 150.6 \mathrm{~Hz}$, and $227.7 \mathrm{~Hz}$, respectively by the selection circuit. The dc gain is declined to $2.9 \mathrm{~dB}$, which demonstrates the non-ideal effect due to the impedance effect.

Fig. 3 (c) shows the group delay characteristics of the low-pass filter. The deviation of the group delay is within the range of $\pm 331.5 \mu$ s up to BW in lowpass filter of $230 \mathrm{~Hz}$ cutoff frequency, $\pm 422 \mu \mathrm{s}$ (in $150 \mathrm{~Hz}$ ), and $\pm 1.132 \mathrm{~ms}$ (in $35 \mathrm{~Hz}$ ). The low-pass filter exhibits a constant group delay response within the prescribed bandwidth, and delay time is only $7 \%$ compared with the period of the signal $\left(f_{\text {signal }}=f_{\text {cutoff } f}\right)$. A transient sinusoidal wave with the amplitude of $200 \mathrm{mVpp}$ is injected into this filter in order to inspect the linearity. Fig. 3 (d) manifests the measured power spectrum of low-pass filter. The HD3 is $-50.7 \mathrm{~dB}$ within the pass-band of $35 \mathrm{~Hz}$. It demonstrates that this biomedical filter is competitive to other designs in biomedical application requiring low-loss dc gain and constant group delay.

\section{Conclusion}

Filters are one of the most important constituent blocks of biomedical recording system. In this paper, we present a reconfigurable fully differential programmable linear-phase filter for bio-signal recording application. A fifthorder ladder-type Bessel Transconductance-Capacitor lowpass filter bas been employed to reduce the influence of coefficient sensitivity and maintain an undistorted bio-signal. Simulation results have shown that linear voltage-tocurrent transconductance combined with current division and sourcedegeneration techniques can be efficiently used for the implementation of low-noise low-distortion OTAs and thus suitable for low-frequency applications. This low-voltage and low-power filter possesses the HD3 of $50.7 \mathrm{~dB}$, dynamic range of $62 \mathrm{~dB}$, dc gain of $-2.899 \mathrm{~dB}$, and power consumption of $30 \mu \mathrm{W}$. Meanwhile, the designed filter can effectively reduce self-adjusting noise on specific channels and anti-aliasing of the analog-to-digital converter. This proves the robustness of the design, even for the application of low voltage low power consumption system is required by the kind of wearable device.

\section{Acknowledgments}

This work was supported by the Fundamental Research Funds for the Central Universities, the Specialized Research Fund for the Doctoral Program of Higher Education, the Ministry of Education, China (20090201120026) and the National Natural Science Foundation of China (61176038 and 61006033). 